\title{
Molecular Identification of Pathogenic Enterococci and Evaluation of Multi-drug Resistance in Enterococcus Species Isolated From Clinical Samples of Some Hospitals in Tehran, Iran
}

\section{Somayyeh saeidi ${ }^{1}$, Reza Mirnejad ${ }^{2 *}$, Sara Masoumi Zavaryani ${ }^{3}$, Shiva $\operatorname{rostamzadeh}^{1}$}

1. Department of Microbiology, Pharmaceutical Sciences branch, Department of Modern Technology, Islamic Azad University, Tehran, Iran

2. Molecular Biology Research Center, Baqiyatallah University of Medical Sciences, Tehran, Iran

3. Department of Microbiology, Varamin-Pishva Branch, Islamic Azad University, Tehran, Iran

\begin{tabular}{c} 
KEYWORDS \\
\hline Enterococcus faecalis \\
Enterococcus faecium \\
Multi-drug Resistance \\
Polymerase Chain Reaction \\
Article Info \\
\hline
\end{tabular}

Received 2018/07/12;

Accepted 2018/09/03;

Published Online 2018

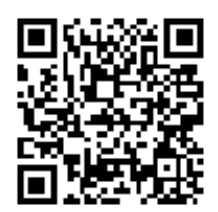

\section{ABSTRACT}

Background and Objectives: Multidrug-resistant (MDR) enterococci cause many problems for physicians and infection control specialists in the recent years. Hence, by identification of antibiotic resistance patterns of enterococci in different geographical regions, an appropriate strategy can be developed to prevent bacterial antibiotic resistance and provide effective treatments. The current study aimed at identifying enterococci via molecular methods and evaluating multi-drug resistance patterns in Enterococcus species isolated from nosocomial samples of some hospitals in Tehran, Iran.

Material and Methods: The current study was conducted on 300 nosocomial samples from different hospitals in Tehran, Iran. The identified Enterococcus species of E. faecalis and E. faecium were isolated via biochemical testing and confirmed using polymerase chain reaction (PCR). The antibiotic resistance pattern was determined using the disc diffusion method according to the Clinical and Laboratory Standards Institute (CLSI) guidelines.

Results: The highest antibiotic resistance was observed against quinupristindalfopristin, tetracycline, and erythromycin. Minimum inhibitory concentration (MIC) of vancomycin against the isolated antibiotic resistant Enterococcus spp. was $\leq 256 \mu \mathrm{g} / \mathrm{mL}$. According to the results of the current study, $69.6 \%$ of E. faecalis and $80 \%$ of E. faecium isolates showed multi-drug resistance.

Conclusion: Increase of antibiotic resistant bacteria, especially MDR species, is a severe health threatening problem worldwide. The increase of MDR bacteria limited the therapeutic solutions to the patients with enterococcal infection, increased treatment costs, and led to transmission of resistant genes among bacteria. It is highly important to find antibiotic resistant patterns to compile guidelines for infectious diseases.

\footnotetext{
Corresponding Information: Reza Mirnejad, Molecular Biology Research Center, Baqiyatallah University of Medical Sciences, Tehran, Iran. Tel/Fax: +98-88039883, E-mail: rmirnejad@bmsu.ac.ir
}

Copyright $\odot$ 2018. This is an open-access article distributed under the terms of the Creative Commons Attribution-noncommercial 4.0 International License which permits copy and redistribute the material just in noncommercial usages, provided the original work is properly cited.

\section{Introduction}

Enterococcus species are considered as one of the main nosocomial pathogens in the recent years. The urinary tract infection followed by pelvic and intra-abdominal infections, bacteremia, endocarditis, etc. are among the most common nosocomial infections caused by enterococci. Such bacteria are the second leading cause of urinary tract infection and the third in nosocomial infections. Enterococcus spp. are Gram-positive cocci and the intestinal flora of human and some animals $(1,2)$. Enterococcus species show poor pathogenicity due to lack of powerful toxins and remarkable virulence factors, but since they are intrinsically insensitive to penicillins, aminoglycosides, and glycopeptides, 
and show high competence in receiving resistance genes; can transfer antibiotic resistance genes to other bacterial genera and species through mutations and/or receiving genetic elements via plasmids or transposons, Enterococcus spp. can cause severe problems in diagnosis, treatment, and control of the associated infections (3).

Nowadays, the multi-drug resistance and insensitivity of such bacteria to antibiotics is of great importance (4). Glycopeptides-resistant Enterococcus spp. are serious and alarming problem worldwide, which affect patients' general health. Glycopeptides such as vancomycin and teicoplanin are the selective and usually the last-resort choice for the hospital-acquired multidrug-resistant (MDR) Gram-positive infections (5). Inappropriate administration and indiscriminate use of broadspectrum beta-lactams, aminoglycosides, and carbapenems as well as long-term hospitalization influence the spread of MDR bacteria (6).

Administration of proper antibiotics should always rely on the antibiotic resistance patterns because the treatment of nosocomial infections caused by such bacteria is very difficult; hence, periodic evaluation of the anti-microbial activity of different antibiotics and understanding the proper drug regimen to treat the infection and control its spread is of great importance because the antibiotic resistance pattern may change within short intervals (7). Transfer of the resistance genes among bacteria via transposons/plasmids, and genetic mutations are among the major causes of the prevalence and spread of antibiotic-resistant bacteria. Since a plasmid or transposon can be the carrier for several resistance elements, resistance to several antimicrobial agents can be acquired simultaneously; it is considered as the reason for the emergence of multidrug-resistant bacteria. Nowadays, increasing multidrug-resistant species and the associated complications are among the great concerns of medical community. Such infections are difficult-to-treat and even in some cases threaten the patients' lives. In addition, due to long-term hospitalization, the treatment is costly; hence, the infections can be significantly controlled and the prevalence can be remarkably limited by the application of cost-effective methods and hospital hygiene standards, as well as microbiologic methods. Identification of Enterococci resistance patterns to commonly used antibiotics to determine therapeutic policy in the initial encounter and effectiveness of controlling programs are essential (8).

In the recent years, molecular techniques such as polymerase chain reaction (PCR) are developed; such techniques are used to identify enterococci as they are very sensitive and benefit from high speed and specificity, compared with the culturing methods (9). Employment of phenotypic methods parallel to genotypic PCR-based methods can provide reliable data. In the current study, both classic and molecular methods were used to identify genus and species. In addition, the frequency, antibiotic resistance pattern, and the prevalence of multidrug-resistance were also studied in Enterococcus species isolated from nosocomial infections in Tehran, Iran.

\section{Materials and methods \\ Sampling}

The current descriptive-cross sectional study was conducted on 300 samples including urinary tract, blood, wound infections, as well as ascites and bronchoalveolar lavage (BAL) collected from Baqiatallah, Milad, and some other hospitals in Tehran, 2015.

\section{Identification of Enterococcus species by phenotypic and genotypic methods}

To identify bacteria isolated from clinical samples, Gram staining method as well as the evaluation of the biochemical characteristics such as catalase reaction, growth in $6.5 \% \mathrm{NaCl}$, and bileesculin hydrolysis were performed. Besides, to identify the bacteria at the species level, the sugar fermentation test (lactose, arabinose, etc.) was conducted using a sugar-based media containing phenol red reagent and incubation at $37^{\circ} \mathrm{C}$ for 24 hours. All isolations were stored at $-70^{\circ} \mathrm{C}$ in brain heart infusion (BHI) broth plus 50\% glycerol until molecular analyses.

Bacterial genome was extracted using boiling methods; then, to confirm E. faecalis and $E$. faecium, PCR was amplified using specific primers. PCR reaction was performed in a total volume of 25 $\mu \mathrm{L}$ containing $1 \mu \mathrm{L}$ DNA pattern $(0.2 \mu \mathrm{g}), 1 \mu \mathrm{L}$ 
each of the primers (10 pmol), $12 \mu \mathrm{L} 2 \mathrm{X}$ Master mix (purchased from Amplicon III, Denmark including $20 \mathrm{mM} \mathrm{dNTP}$ and $1.5 \mathrm{mM} \mathrm{MgCl}_{2}$ ), and $11 \mu \mathrm{L}$ double distilled water. Then, the following PCR program was set using a Eppendorf thermocycler (Hamburg; Germany): early denaturation for 1 minute at $94^{\circ} \mathrm{C}, 35$ cycles of denaturation for 1 minute at $94^{\circ} \mathrm{C}$, and annealing for 1 minute at $55^{\circ} \mathrm{C}$ and early extension for 2 minutes at $72^{\circ} \mathrm{C}$. The final extension was done for 5 minutes at $72^{\circ} \mathrm{C}$. The PCR product was electrophoresed on $1.5 \%$ gel agarose in a Gel Documentation (Table 1).

Table 1. The Primer Sequences Used for PCR Amplification to Identify Enterococcus faecalis and Enterococcus faecium

\begin{tabular}{cccc}
\hline Primer & Primer Sequence & Size of the & Reference \\
& & Fragment & \\
\hline ddl $\boldsymbol{E}$. faecalis & F- 5'ATCAAGTACAGTTAGTCT 3' & $941 \mathrm{bp}$ & 10 \\
\hline ddl $\boldsymbol{E}$. faecium & R- 5'ACGATTCAAAGCTAACTG 3' & & 10 \\
\hline & F- 5'TAGAGACATTGAATATGCC 3' & $550 \mathrm{bp}$ & \\
\hline
\end{tabular}

\section{Antibiotic profile}

The disk diffusion method was used to determine the antibiotic sensitivity pattern of the isolates on Muller-Hinton Agar (Merck), according to the Clinical and Laboratory Standards Institute (CLSI) 2015 guidelines; results were reported as resistant, semi-sensitive, and sensitive for each antibiotic (11).

Vancomycin $30 \mu \mathrm{g}$, erythromycin $15 \mu \mathrm{g}$, quinupristin / dalfopristin $15 \mu \mathrm{g}$, tetracycline $30 \mu \mathrm{g}$, ciprofloxacin $5 \mu \mathrm{g}$, teicoplanin $30 \mu \mathrm{g}$, linzolaide 30 $\mu \mathrm{g}$, fosfomycin $200 \mu \mathrm{g}$, chloramphenicol $30 \mu \mathrm{g}$, gentamicin $120 \mu \mathrm{g}$, ampicillin $10 \mu \mathrm{g}$, nitrofurantoin and $300 \mu \mathrm{g}$ (Mast; UK), as well as E. faecalis ATCC29212 and E. faecium BM 4147 were used as the quality control species in the current study.
The minimum inhibitory concentration (MIC) for the isolates resistant to vancomycin was conducted based on the microdilution method. The isolation showed resistance to 3 or more antibiotic lines were considered as MDR species.

\section{Results}

Out of 300 samples collected in the current study, 140 enterococcus species were identified, which constituted $68 \%$ of all urinary tract isolated species (Table 2).

Results of the phenotypic- based identification methods were confirmed by PCR amplification using specific primers; accordingly, out of 140 identified species $125(89.28 \%)$ were E. faecalis and $15(10.72 \%)$ E. faecium (Figure 1).

Table 2. The Frequency Percentage of Enterococcus faecalis and Enterococcus faecium Species Isolated from Nosocomial Samples in the Current Study

\begin{tabular}{cccc}
\hline Sample Type & $\begin{array}{c}\text { Isolated Enterococci } \\
(\mathbf{N = 1 4 0 )}\end{array}$ & $\begin{array}{c}\text { Isolated E. faecalis, } \mathbf{N}(\boldsymbol{\%}) \text { Isolated E. faecium, } \mathbf{N}(\%) \\
(\mathbf{N}=\mathbf{1 5})\end{array}$ \\
\hline Urinary tract & 92 & $85(68)$ & $7(46.7)$ \\
\hline Wound & 24 & $21(16.8)$ & $3(20)$ \\
\hline Blood & 17 & $14(11.2)$ & $3(20)$ \\
\hline BAL & 2 & 0 & $2(13.3)$ \\
\hline Other & 5 & $5(4)$ & 0 \\
\hline
\end{tabular}




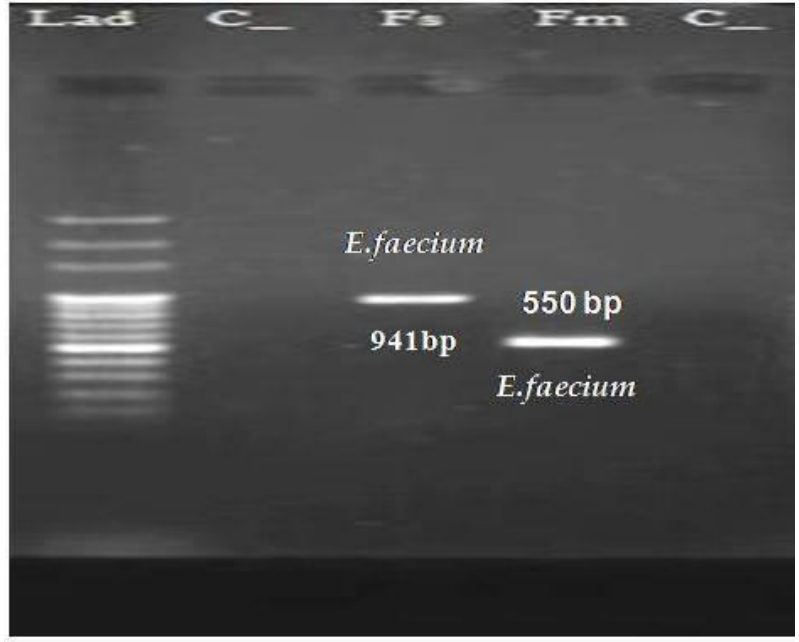

Figure 1. PCR products; Lane 1) DNA marker 100 bp; Lane 2) The amplicon without the targeted gene in the clinical isolations of E. faecium; Lane 3) The amplicon including $d d l$ gene $(550 \mathrm{bp})$ in the clinical isolation of $E$. faecium; Lane 4) The amplicon including $d d l$ gene (941 bp) in the clinical isolations of E. faecalis; Lane 5) The amplicon without targeted gene in the clinical isolations of E. faecalis

\section{Antibiotic susceptibility testing}

The highest antibiotic resistance in the studied isolates was observed against quinupristin / dalfopristin, followed by tetracycline and erythromycin. Results of antibiogram testing for the current study isolates, according to CLSI guidelines, are shown in Table 3.

Table 3. Results of Antibiotic Susceptibility Testing for the Study Isolates, Based on the Disc Diffusion Method

\begin{tabular}{|c|c|c|c|c|c|c|c|}
\hline \multirow[t]{2}{*}{ Antibiotic } & \multicolumn{3}{|c|}{ Enterococcus faecalis } & \multicolumn{4}{|c|}{ Enterococcus faecium } \\
\hline & $\begin{array}{l}\text { Sensitive, } N \\
(\%)\end{array}$ & $\begin{array}{c}\text { Semi- } \\
\text { lsitive, } \mathrm{N}(\%)\end{array}$ & $\begin{array}{l}\text { Resistant, } \mathbf{N} \\
\text { (\%) }\end{array}$ & $\begin{array}{l}\text { Sensitive, } N \\
(\%)\end{array}$ & $\begin{array}{l}\text { Semi-sensitive } \\
\mathbf{N}(\%)\end{array}$ & Resis & nt, $\mathbf{N}(\%)$ \\
\hline Tetracyclin & $23(18.88)$ & 0 & $102(81.12)$ & $4(26.68)$ & 0 & & 73.32) \\
\hline Gentamicin & $80(64.51)$ & $23(18.29)$ & $22(17)$ & $9(60)$ & $4(26.8)$ & & 13.2) \\
\hline Ampicillin & $115(92)$ & 0 & $10(8)$ & $9(60)$ & 0 & & $(40)$ \\
\hline Vancomycin & 113(90.04) & $9(6.8)$ & $4(3.16)$ & $10(66.8)$ & $1(6.52)$ & & 26.68) \\
\hline Fosfomycin & & $25(20)$ & $47(37.45)$ & $53(42.55)$ & $6(40)$ & $5(33.32)$ & $4(26.68)$ \\
\hline Linzeolid & & $116(92.8)$ & $8(6.27)$ & $1(0.08)$ & $14(93.48)$ & 0 & $1(6.52)$ \\
\hline Nitrofurantoin & & $120(96)$ & $3(2.4)$ & $2(1.6)$ & $10(66.8)$ & $2(13.2)$ & $3(20)$ \\
\hline Ciprofloxacin & & $38(30.04)$ & $44(35.65)$ & $43(34.4)$ & $3(20)$ & 0 & $12(80)$ \\
\hline Erythromaycin & & $19(15.2)$ & $37(29.6)$ & $69(55.2)$ & $1(6.52)$ & $1(6.52)$ & 13(86.96) \\
\hline Teicoplanin & & $119(95.2)$ & $4(3.51)$ & $2(1.29)$ & $10(66.8)$ & 0 & $5(33.2)$ \\
\hline Chloramphenicol & & $85(68)$ & $21(16.8)$ & 19(15.2) & $4(26.68)$ & $3(20)$ & $8(53.32)$ \\
\hline Quinpristin/dalfopristin & & $11(8.8)$ & $5(4)$ & $109(87.2)$ & $4(26.68)$ & $3(20)$ & $8(53.32)$ \\
\hline
\end{tabular}

Table 4. The Frequency of Multidrug-resistant Enterococcus faecalis and Enterococcus faecium Species Isolated in the Current Study

\begin{tabular}{|c|c|c|c|c|c|}
\hline \multicolumn{6}{|c|}{ Number of the Isolates Resistant to One or More Antibiotics } \\
\hline $\begin{array}{l}\text { Num } \\
\text { the Ant }\end{array}$ & 1 & 2 & 3 & 4 & $>4$ \\
\hline E. faecalis & 12 & 27 & 28 & 21 & 37 \\
\hline E. faecium & 2 & 1 & 2 & 1 & 9 \\
\hline
\end{tabular}

In the current study, $3.16 \%$ of E. faecalis and $26.68 \%$ of $E$. faecium species showed resistance to vancomycin. MIC of the isolated species to vancomycin was $\geq 256 \mu \mathrm{g} / \mathrm{mL}$. All vancomycinresistant isolates showed MDR; in such a way that 1 vancomycin-resistant $E$. faecalis isolate showed resistance to 4 other antibiotics, $1 \mathrm{E}$. faecium isolate was resistant to 12 antibiotics, 1 isolates of $E$. faecalis was resistant to 7 antibiotics, 1 isolate of $E$. faecalis was resistant to 9 antibiotics, 3 isolates of $E$. faecium were resistant to 8 antibiotics, and $2 E$. faecalis isolates showed resistance to 6 antibiotics.

\section{Discussion}

During the recent years, the growing prevalence of enterococci infections with antibiotic resistance caused severe and significant complications in the health care system of different countries (12). The 
researchers showed that although the level of general health enhanced in the communities, the bacterial infections, particularly enterococcal infections, increase, which mainly results from intrinsic resistance of Enterococcus spp. to antibiotics and the linear transfer of resistant genes, transposons, and plasmids among enterococci and/or other bacterial genera. Besides, indiscriminate and improper administration of antibiotics is another reason for the spread of antibiotic resistance in different geographical regions. Uncontrolled consumption of broadspectrum beta-lactams as well as long-term hospitalization influenced the spread of multidrugresistant bacteria; the treatment of such infections is difficult and may leads to mortality $(3,5,6)$.

Enterococcus faecalis is the most Enterococcus species isolated from clinical samples in the majority of the studies. In the current study, out of 140 isolated enterococci, $125(89.28 \%)$ were $E$. faecalis and $15(10.72 \%)$ E. faecium. In the studies by Dadfarma, Kafil, and Comerlato, most of the clinically isolated species were E. faecalis, consistent with the results of the current study (1315). However, in another study by Bibalan and Oskuie, most of the isolated enterococci were $E$. faecium, inconsistent with the results of the current study; the difference between the results may be attributed to the type of samples and the type of commonly administered antibiotics $(16,17)$. In the current study, similar to those of Padmasini and Behnood, most of the enterococcal species were isolated from urinary tract samples. The dominance of enterococci among the species isolated from urinary tract samples is another reason for the prevalence of enterococci in the urinary tract infections. The bacterial multidrug-resistance has various frequencies in different countries, which may be attributed to genetic changes in bacterial species, different normal flora, and different antibiotic prescription patterns, as well as different rates of antibiotic consumption in the communities $(18,19)$.

In the current study, E. faecalis isolates showed the highest resistance to tetracycline, quinupristin / dalfopristin, erythromycin, and fosfomycin, respectively. In addition, E. faecium isolated species were mostly resistant to tetracycline, ciprofloxacin, erythromycin, and quinupristin / dalfopristin. The results of the current study were similar to those of Seifi and Talebi $(20,21)$.

In the current study, out of 140 enterococci isolates, 2 isolates (E. faecalis and E. faecium) were resistant to linzolaide, similar to the results of Yaslani and Bhatt $(22,23)$. But, in the studies by Kafil Bibalan, Mahesh, and Wang, all isolated species were sensitive to linzolaide; the difference can be attributed to the type of study, geographical region, type of antibiotic, and the employed methods $(14,16,24,25)$. The resistance to gentamicin and ampicillin in the current study was similar to the results of Tabatabaei and in contradiction with the results of Yadegarynia $(26,27)$.

According to the results of the current study, resistance to vancomycin in $E$. faecalis and $E$. faecium was $3.16 \%$ and $26.68 \%$, respectively. In the studies by Sanal and Tabatabaei, most of the species were sensitive to vancomycin $(26,28)$. In addition, studies by Hoseini Zadeh and Kafil reported higher levels of resistance to vancomycin, compared with the current study $(14,29)$.

The emergence of multidrug-resistant Enterococcus spp. is critically important and 69.6\% of E. faecalis and $80 \%$ of E. faecium species isolated in the current study showed multidrug-resistance. In the studies by Bibalan and Aleyasin, the frequency of MDR species was lower than that of the current study (16 vs. 2 MDR species) $(36,16)$. Results of the studies by Wang et al. and Sharifi et al., showed that more than $80 \%$ and $79 \%$ of the enterococci isolates were MDR, respectively $(15,31)$. In addition, results of the studies by Dadfarma, Behnood, and Deshpande reported 45.7\%, 49.59\%, and $57 \%$ of the isolated enterococci as MDR, respectively $(14,19,32)$.

Also, results of the studies by Telkar, Mira, and Mahesh reported the prevalence of MDR species $(24,33,34)$.

\section{Conclusion}

Results of the current study indicated that $E$. faecalis was the most common isolated species in the clinical samples. The majority of enterococcal species were isolated from urinary tract samples. In addition, results of the current study showed that the isolated species of E. faecalis were more resistant 
against tetracycline, quinupristin / dalfopristin, erythromycin, and fosfomycin, but E. faecium showed more resistance against tetracycline, ciprofloxacin, erythromycin, and quinupristin / dalfopristin. Most of the isolated Enterococcus spp. were resistant against 3 or more antibiotics and accordingly were considered as MDR bacteria. Hence, administration of proper antibiotics based on the results of timely and meticulous antibiogram plays a significant role in the treatment and prevention of the MDR bacteria spread. Accordingly, it is recommended to compile more detailed and distinct instructions for more logical administration of antimicrobial agents and prevent infections, particularly nosocomial infections.

\section{Acknowledgments}

The current study was conducted in the Molecular Biology Research Center of Baquatallah University of Medical Sciences. The authors acknowledge their gratitude to the experts and staff of the center for their cooperation with the study.

\section{References}

1. Bourdon N, Fines-Guyon M, Thiolet JM,Maugat S, Coignard B, Leclercq R, et al.Changing trends in vancomycin-resistant enterococci in French hospitals, 2001-08. JAntimicrob Chemother 2011; 66(4): 713-721.

2. Fisher K, Phillips C. The ecology epidemiology and virulence of Enterococcus, Microbiology. Microbiology. 2009;155(Pt 6):1749-57

3. Simjee S, White DG, McDermott PF, Wagner DD, Zervos MJ, Donabedian SM, English LL, Hayes JR, Walker RD.Characterization of Tn1546 in vancomycinresistant Enterococcus faecium isolated from canine urinary tract infections: evidence of gene exchange between human and animal enterococci. J Clin Microbiol. 2002;40(12):4659-65.

4. Alexander J , Adam Mc. Antibiotic Resistance, How Serious is the Problem and what can be done. Clinical Chem. 2012; 58: 11821186
5. Woodford N. Biological counterstrike:antibiotic resistance mechanisms of Grampositive cocci. Clinical Microbiology andInfection. 2005; 11: 2-21.

6. Zavascki AP, Carvalhaes CG, Piccaso RC, Gales AC. Multidrug-resistant Pseudomonas aeruginosa and Acinetobacter baumannii: resistance mechanisms and implications for therapy. Expert Rev Anti Infect Ther 2010; 8(1):71-93.

7. Kalantar E, Motlagh ME, Lornejad H, Reshadmanesh N.Prevalence of urinary tract pathogens and antimicrobial susceptibility patterns in children at hospitals in Iran.Iranian Journal of Clinical Infectious Diseases. 2008; 3(3):149-153.

8. Eriksen H.M, Iversen B.G, Aavitsland P. Prevalence of nosocomial infections in hospital in Norway, 2002 and 2003. J Hosp infec. 2005, 60: 40-45

9. Preethee TH, Kandaswamy D, Hannah R. Molecular identification of an Enterococcus faecalis endocarditis antigen efaA in root canals of therapy-resistant endodontic infections. $\mathrm{J}$ Conserv Dent. 2012; 15(4): 319-322.

10. Ross JE., Scangarella-Oman NE., Flamm RK., Jones RN. Determination of disk diffusion and MIC quality control guidelines for GSK2140944, a novel bacterial type II topoisomerase inhibitor antimicrobial agent. J. Clin. Microbiol. 2014;52: 2629-2632,

11. Clinical and Laboratory Standards Institute Performance Standards for Antimicrobial Susceptibility Testing; TwentyThird Informational Supplement. CLSI document M100-S25. Wayne, PA: Clin Labora Stand Institu; 2015

12. Mundy L, Sahm D, Gilmore M. Relationships between enterococcal virulence andantimicrobial resistance. Clin Microbiol Rev. 2000; 13:513-522.

13. Dadfarma N, Imani Fooladi A.A, Oskoui M, Mahmoodzadeh Hosseini H. High level of gentamicin resistance (HLGR) among enterococcus strains isolated from clinical 
specimens. J. Infect. Public Health. 2013;6, 202208.

14. Kafil HS, Asgharzadeh M, Vancomycin-resistant enteroccus faecium and enterococcus faecalis isolated from education hospital of iran. Maedica (Buchar). 2014;9(4):323-327

15. Comerlato $\mathrm{CB}$, Resende $\mathrm{MC}$, Caierão $\mathrm{J}$, d'Azevedo PA. Presence of virulence factors in Enterococcus faecalis and Enterococcus faecium susceptible and resistant to vancomycin. Mem Inst Oswaldo Cruz. 2013;108(5):590-5.

16. Hasannejad Bibalan M, Eshaghi M, Sadeghi J, Asadian M, Narimani T, Talebi M. Clonal Diversity in Multi Drug Resistant (MDR) Enterococci Isolated from Fecal Normal Flora. Int J Mol Cell Med. 2015; 4(4): 240-244

17. Oskoui M, Farrokh P. Distribution of insertion sequences associated with Tn1546 and clonal diversity of vancomycin-resistant enterococci isolated from patients in Tehran, Iran .IJM.2010; 2 (1): 14-21

18.Padmasini E., Padmaraj R., Ramesh S.S. High level aminoglycoside resistance and distribution of aminoglycoside resistant genes among clinical isolates of Enterococcus species in Chennai, India. Scientific World Journal. 2014;329157:1-5

19. Behnood A, Farajnia S, Moaddab SR, Ahdi-Khosroshahi S, Katayounzadeh A. Prevalence of aac(6')-Ieaph(2')-Ia resistance gene and its linkage to Tn5281 in Enterococcus faecalis and Enterococcus faecium isolates from Tabriz hospitals. Iran J. Microbiol. 2013;(5) :203-208

20. Saifi M, Pourshafie MR, Eshraghian MR, Soltan Dallal MM. Anti-Microbial Resistance of Enterococci Isolated from Urinary Tract Infections in Iran. Iran Biomed J. 2008;12(3):185-90.

21.Talebi M, Eshraghi SS, Pourshafie MR, Pourmand MR, Eshraghian MR. Characterization of Vancomycin Resistant Enterococcus faecium, Iranian $\mathrm{J}$ Publ Health. 2007;36;(4),:20-25
22. Yasliani S, Mohabati Mobarez A, Hosseini Doust R, Satari M, Teymornejad O.. Linezolid vancomycin resistant Enterococcus isolated from clinical samples in Tehran hospitals. Indian J Med Sci. 2009;63(7):297-302

23.Puneet Bhatt M, Patel A, Brig A.K. Sahni c,Surg Cmde A.K. Praharaj,et.al. Emergence of multidrug resistant enterococciat a tertiary care centrem . Med J Armed Forces India. 2015; 71(2): 139-144.

24. C. Baragundi Mahesh, Sonth S.B, Solabannavar S.S, Yemul V. Species Prevalence And Antimicrobial Resistance Pattern Of Enterococcal Isolates In A Tertiary Health Care Centre Journal of Clinical and Diagnostic Research.2010; 40(6):3405-3409

25. Wang JT , Chang SC , Wang HY, Chen PC, Shiau YR, Lauderdale TL, TSAR Hospitals . High rates of multidrug resistance in Enterococcus faecalis and E. faecium isolated from inpatients and outpatients in Taiwan.Diagnostic Microbiology and Infectious Disease. 2013, 75(4),: 406-411

26. Rafiei S, Tabatabaei, A, Karimi M, avidinia M, Fallah F, Tavakkoly Fard A, Rahbar M. A study on prevalence of vancomycinresistant enterococci carriers admitted in a children hospital in Iran. Annals of Biological Research.2012, 3 (12):5441-5445

27.Davood Yadegarynia, Latif Gachkar, Jalal Karimi, Alireza Mazloum Fazel, Alireza Fatemi, Zeinab Afzalvatan Naeini. The Pattern of Antimicrobial Resistance of Enterococci: A Hospital-Based Study in Iran. Int. J. Rev. LifeSci,2015, 5(10), 351-354

28. Sanal C. Fernandes. B. Dhanashree. Drug resistance \& virulence determinants in clinical isolatesof Enterococcus species. Indian J Med Res. 2013:13(7): 981-985

29. Hoseini Zadeh A; Shojapour M; Nazari R; Akbari M; SofianM; Abtahi H. Genotyping of Vancomycin Resistant Enterococci in Arak Hospitals. Jundishapur $\mathbf{J}$ Microbiol. 2015;.8(4); 
30. Aleyasin, A.M. Mobarez, M. Sadeghizadeh, R. Hosseini Doust, N. Khoramabadi. Resistant to Vancomycin in Enterococcus faecium and faecalis clinical isolatesI. Pak J Med Sci. 2007; 23( 3): 390-393

31. Sharifi Y, Hasani A, Ghotaslou R, Naghili B, Aghazadeh M, Milani M, Bazmani A. Virulence and Antimicrobial Resistance in Enterococci Isolated from Urinary Tract Infections. Advanced Pharmaceutical Bulletin. 2013; 3(1): 197-201

32. Deshpande R, Karmarkar MG, Mehta PR. Prevalence of multidrug-resistant enterococci in a tertiary care hospital in Mumbai, India. J Infect Dev Ctries. 2013; 7(2):155-158
33. Mira MU, Deana M, Zora J, Vera G, BiljanaM , Biljana R. Prevalence of different enterococcal species isolated from blood and their susceptibility to antimicrobial drugs in Vojvodina, Serbia, 2011-2013. 2014 ; 8(8): 819824.

34. Telkar A, Mahesh B. 405 in the Prevalence and the Antibiotic Resistance of the Enterococcal Species Isolated from Blood Cultures .Journal of Clinical and Diagnostic Research. $2012 ; 6(3): 405-407$

\section{How to Cite This Article:}

Saeidi S, Mirnejad R, Masoumi Zavariani S, Rostasmzadeh S. Molecular Identification of Pathogenic Enterococci and Evaluation of Multi-drug Resistance in Enterococcus Species Isolated From Clinical samples of Some Hospitals in Tehran, Iran. Mod Med Lab J. 2018; 1 (2) :60-67 